\title{
Photophysics of the LOV-Based Fluorescent Protein Variant iLOV-Q489K Determined by Simulation and Experiment
}

Mehdi D. Davari, ${ }^{*, \dagger}$ Benita Kopka, ${ }^{\ddagger}$ Marcus Wingen, ${ }^{\ddagger}$ Marco Bocola, $\uparrow$ Thomas Drepper, ${ }^{\ddagger}$ Karl-Erich Jaeger, ${ }^{\ddagger, \S}$ Ulrich Schwaneberg, ${ }^{\dagger}, \|$ and Ulrich Krauss ${ }^{\star},{ }^{\ddagger}$

$\dagger$ Lehrstuhl für Biotechnologie, RWTH Aachen University, Aachen, Germany $\$$ Institut für Molekulare Enzymtechnologie, Heinrich Heine University

Düsseldorf, Forschungszentrum Jülich, Jülich, Germany § Institut für Bio- und Geowissenschaften, IBG-1: Biotechnologie, Forschungszentrum Jülich, 52426 Jülich, Germany ॥DWI-Leibniz Institute for Interactive Materials, Forckenbeckstraße 50, 52056, Aachen, Germany

${ }^{*}$ Corresponding author

Email: mehdi.davari@rwth-aachen.de and u.krauss@fz-juelich.de 


\section{Analysis of electron density maps of crystal structures of parental iLOV}

Electron density maps (2Fo-Fc) of the crystal structures of parental iLOV (PDBcodes $4 \mathrm{EES}$ and $4 \mathrm{EET}$ ) were obtained from the Electron Density Server at Uppsala University ${ }^{1}$ (http://eds.bmc.uu.se/eds/) and visualized by Pymol. ${ }^{2}$ The resolution of the two structures (1.8 and $1.2 \AA$, respectively) is different but the Q489 side-chain shows well-defined electron density in both 4EES and 4EET as shown in Figure S1. The overlay of the structures shows that orientation of Q489 in both structures is similar. The occupancies of Q489 are 1 and we don't observe a reduced electron density for the side chain of this residue in the electron density maps (Shown in Figure S1) of both structures. 
(a)

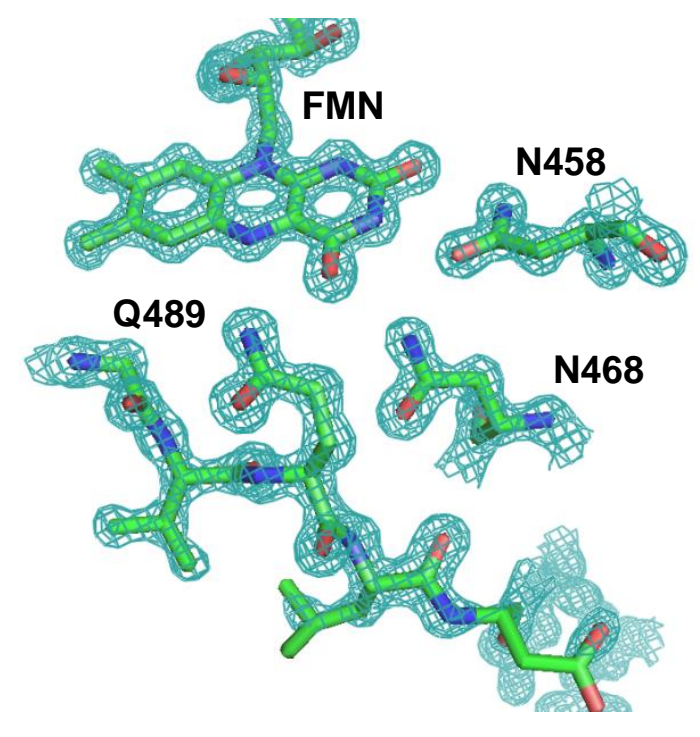

(b)

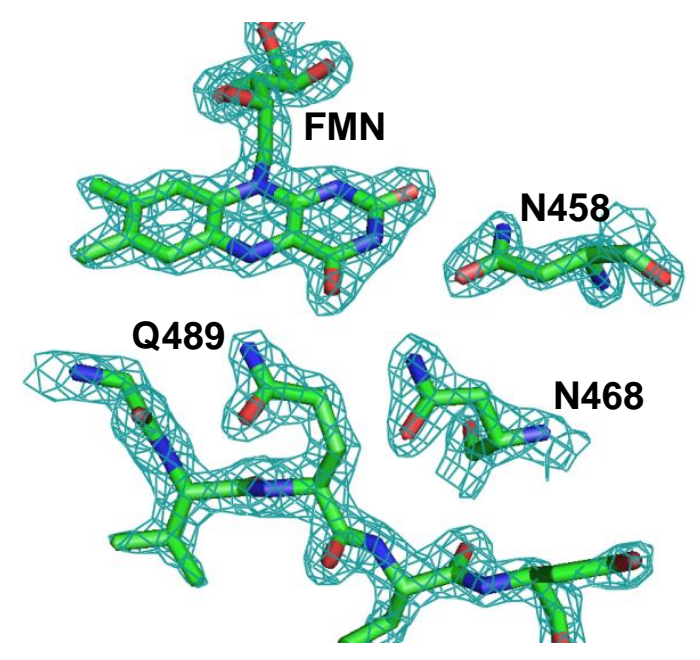

Figure S1: Electron density map (2Fo-Fc contoured at 2.0 sigma) of crystal structures of parental iLOV ${ }^{3}$ (a) PDB-code 4EET and (b) 4EES, resolution 1.2 and $1.8 \AA$, respectively. Side-chain orientations of Q489, N468, N458 and FMN are shown in figure in stick representation. Electron density map of the iLOV Xray crystal structure shows a well-defined electron density for the side chain of Q489 both in 4EES and 4EET. 


\section{Analysis of FoldX calculations}

Starting from the crystal structure of parental iLOV (PDB-code: 4EES ${ }^{3}$ ), the iLOV-Q489 single mutant was built by using the FoldX ${ }^{4}$ method after repairing the structure using RepairPDB command. 10 independent runs for rotamer searches employing a probability-based rotamer library were performed to predict the best rotamer while exploring alternative conformations of the surrounding side chains below $6 \AA$ distance from Q489. In the FoldX calculations residue backbones are kept rigid. Figure $\mathrm{S} 2$ visualizes the spatial overlapping of K489 orientations in iLOV-Q489 variant predicted by FoldX analysis (residue K489 is shown as stick). The conformers mainly differ in the terminal side chain dihedral angle (i.e. NZ-CE-CD-CG) of K489. Table S1 lists this dihedral angle and the distance between K489-NZ and FMN-O4 atoms in 10 independent runs of the iLOV-Q489K variant generated by using the FoldX method. A detailed analysis of Q489 substitution in iLOV regarding stabilization energy is shown in Figure $\mathrm{S} 3$. Calculated stabilization energy $(\Delta \Delta \mathrm{G})$ corresponds to the Gibbs free energy changes upon substitution of Q489 to K489 in unfolded and folded states compared to parental iLOV. Basically, the more stabilizing conformer gives a larger negative value for mutational energy, $(\Delta \Delta \mathrm{G})$, (standard deviation of FoldX calculations is $0.81 \mathrm{kcal} / \mathrm{mol}{ }^{4}$ ). Results shown in Figure 3 revealed that the three best conformers (oriented towards Gly487) are having a slight destabilizing effect (values less than cutoff 1.5 $\mathrm{kcal} / \mathrm{mol}$ are considered as stable substitution). In contrast, the remaining conformers showed a more destabilizing effect of about $2.5 \mathrm{kcal} / \mathrm{mol}$ and most of them are pointing towards the $\mathrm{O}$ atom of $\mathrm{G} 487$ backbone.

For iLOV-Q489K, two distinct orientations of K489 are observed as shown in Figure S2 (denoted as $\mathrm{K}_{489}$ in and $\mathrm{K} 489_{\text {out }}$ ). In conformers occupying the socalled $\mathrm{K} 489_{\text {in }}$ position there is a hydrogen bond between NZ of K489 and FMNO4, whereas a hydrogen bond is formed between backbone oxygen of G487 (G487-O) in conformers occupying so-called K489 out position. As can be seen in Figure S2, run number 6 occupies the $K 489_{\text {out }}$ position oriented towards the backbone of G487. Because in the FoldX method backbone atoms are kept 
fixed during the rotamer search procedure, the $\mathrm{K} 489_{\text {out }}$ conformer found in run number 6 gives rise to lower stabilization energy with respect to run number 1 in $\mathrm{K} 489_{\text {in }}$ position, indicative of the steric clashes of sidechain of the K489 side chain and the G487 backbone. From this analysis we have selected FoldX run number 1 as representative for the $\mathrm{K} 489_{\text {in }}$ conformer for further refinement by using MD simulations.

In addition, the FoldX method provides a quantitative estimation of the interactions between the FMN and FMN binding pocket upon mutation. The distribution of the interaction energy between FMN and binding pocket for 10 runs is shown in Figure S4. Calculated interaction energies suggest that in most of runs the interactions of the mutant residue are within $1 \mathrm{kcal} / \mathrm{mol}$ the same as in parental iLOV, among which run number 1 (K489 $9_{\text {in }}$ position ) has a more favorable interaction energy than run number 6 (K489 ${ }_{\text {out }}$ position). 


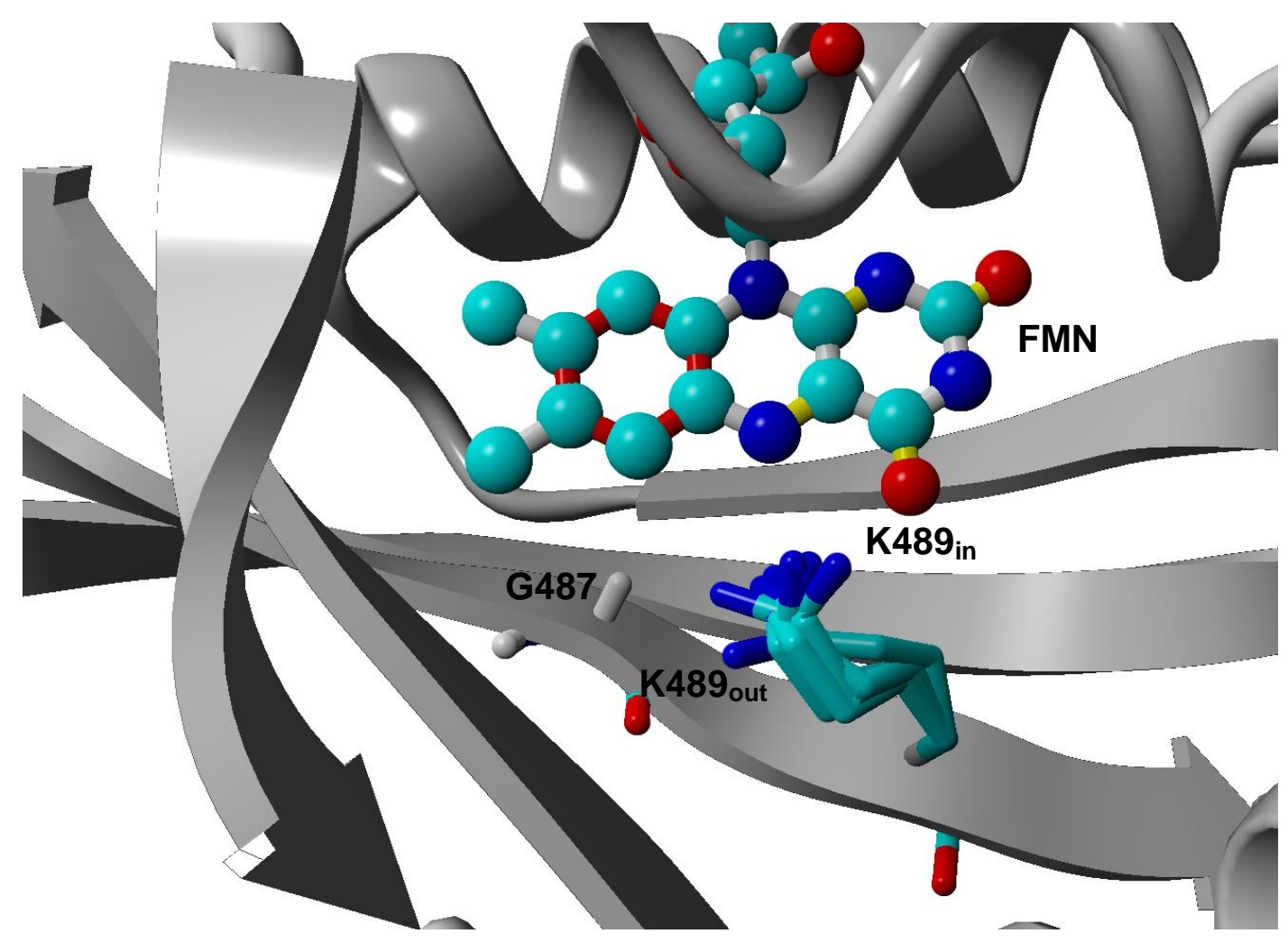

Figure S2: K489 side-chain orientations in 10 independent FoldX runs of iLOVQ489K variant. FMN is shown in ball-stick with carbon (cyan), oxygen (red), nitrogen (blue), and phosphorous (yellow); 10 conformers of K489 are shown as stick. Two distinct conformers of K489 are seen (i.e. K489 in and $K 489_{\text {out }}$ ). In conformers occupying the $\mathrm{K} 489_{\text {in }}$ position there is a hydrogen bond between FMN-O4 and the K489-NZ atom, whereas a hydrogen bond is formed between the side chain amino group of K489 and G487-O in conformers occupying K489 ${ }_{\text {out }}$ position. Different conformers of K489 have different terminal sidechain dihedral angles (NZ-CE-CD-CG). 
Table S1: Terminal sidechain dihedral angle (NZ-CE-CD-CG) of K489 and the distance between K489-NZ and FMN-O4 atoms in 10 independent runs of iLOV-Q489K variant generated by using the FoldX method.

\begin{tabular}{ccc}
\hline run & $\begin{array}{c}\text { dihedral angle } \\
\text { NZ-CE-CD-CG }\left(^{\circ}\right)\end{array}$ & $\begin{array}{c}\text { distance } \\
\text { K489-NZ } \cdots \text { FMN-O4 (Å) }\end{array}$ \\
\hline $\mathbf{1}$ & 65 & 3.21 \\
$\mathbf{3}$ & 53 & 3.24 \\
$\mathbf{4}$ & -63 & 2.60 \\
$\mathbf{5}$ & 162 & 4.34 \\
$\mathbf{6}$ & 130 & 3.86 \\
$\mathbf{7}$ & 61 & 2.81 \\
$\mathbf{8}$ & 53 & 2.92 \\
$\mathbf{9}$ & -100 & 2.89 \\
$\mathbf{1 0}$ & -77 & 2.69 \\
\hline
\end{tabular}




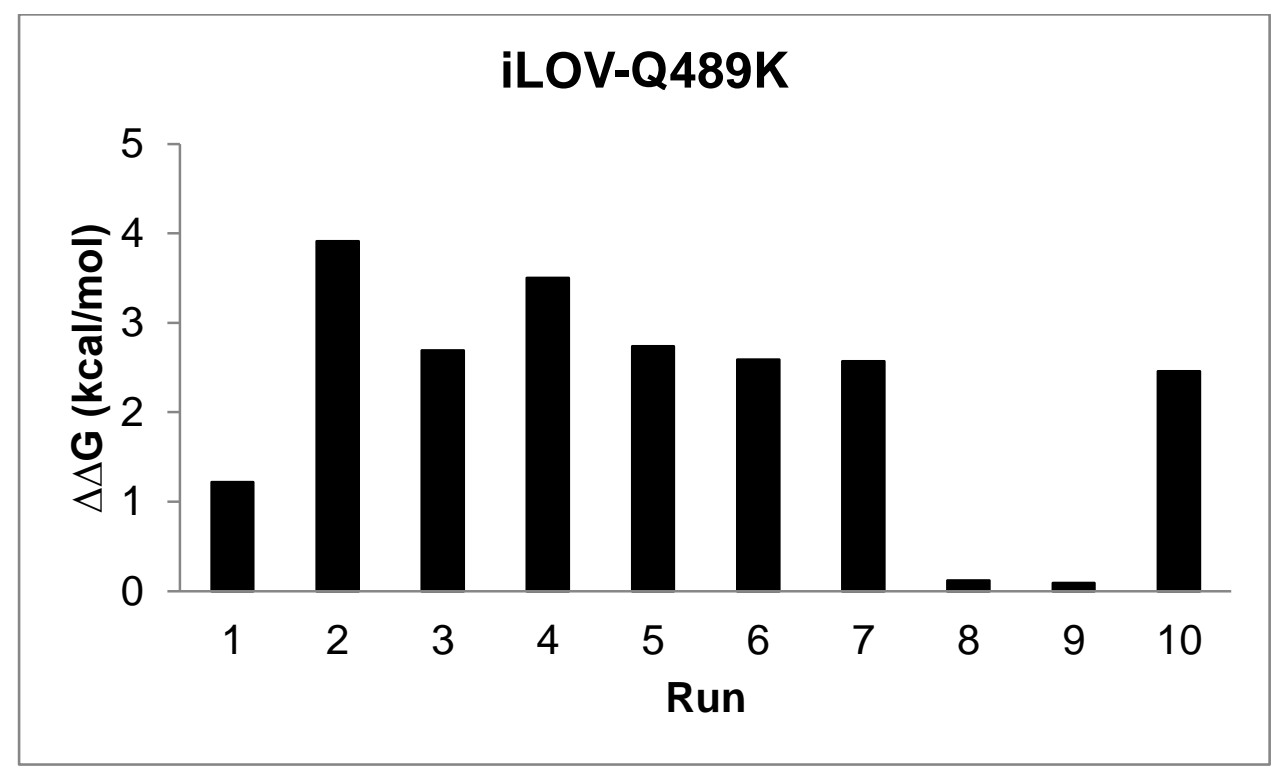

Figure S3: Calculated stabilization energies $(\Delta \Delta \mathrm{G})$ in $\mathrm{kcal} / \mathrm{mol}$ for 10 independent runs of iLOV-Q489K variant with respect to parental iLOV by using the FoldX method; $\Delta \Delta G=\Delta G$ (mutant) $-\Delta G$ (parental) .

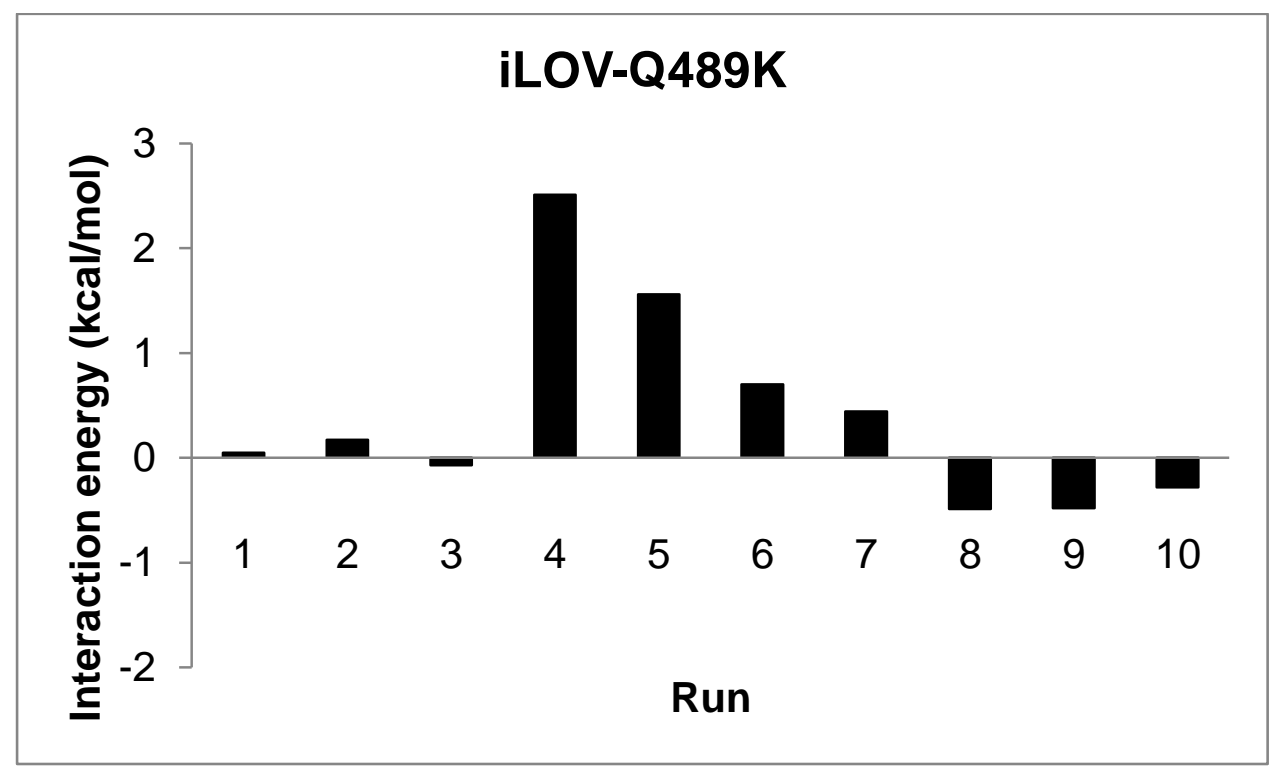

Figure S4: Calculated interaction energy $(\Delta \Delta \mathrm{G}$ in $\mathrm{kcal} / \mathrm{mol})$ between $\mathrm{FMN}$ and FMN binding pocket (protein) for 10 independent runs of iLOV-Q489K compared to parental iLOV by using the FoldX method; $\Delta \Delta G=\Delta G$ (mutant) $\Delta \mathrm{G}$ (parental). 


\section{Analysis of MD Simulations}

Molecular dynamics (MD) simulation for parental iLOV and the iLOV-Q489K variant were run for $50 \mathrm{~ns}$ in triplicate (run_1, run_2, run_3). Pymol ${ }^{5}$ and VMD ${ }^{6}$ were used for molecular visualizations and cpptraj in AmberTool $14^{7}$ was used for distance and hydrogen bond analysis. In order to monitor the flipping of Q489 and K489 along trajectories we analyzed the distance between FMN-O4 *. Q489-NE2, FMN-N5 ‥ Q489-NE2 in parental iLOV and FMN-O4 $\cdots$ K489-NZ, FMN-N5 $\cdots$ K489-NZ, and K489-NZ $\cdots$ G487-O in iLOV-Q489K as shown in Figures S6-S9, respectively. In ILOV-Q489K simulations when starting from the

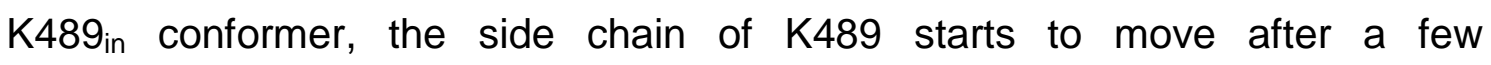
nanoseconds towards the G487 thus populating the K489 ${ }_{\text {out }}$ conformer (shown in Figure S8 and Figure S9), which strongly suggest that the flipping of K489 towards G487 provides sufficient space for the large K489 next to the FMN chromophore.

Time evolution of hydrogen bonds between FMN-O4, FMN-N5, Q489-NE2, K489-NZ and G487-O were analyzed for parental iLOV and iLOV-Q489K variant, respectively. Geometric cutoff for hydrogen bond distance and angle were considered $3.2 \AA$ and $150^{\circ}$, respectively. We analyzed the population of respective hydrogen bonds to monitor the flipping of K489. Hydrogen bond analysis depicted in Table S3 in parental iLOV shows that the $\mathrm{H}$-bond between FMN-O4 and Q489-NE2 is persistent during the trajectories while no hydrogen bond is observed between FMN-N5 and Q489-NE2 in parental iLOV. Additionally, hydrogen bond analysis in the iLOV-Q489K variant shows that the H-bond between FMN-O4 ‥ K489-NZ, FMN-N5 $\cdots$ K489-NZ breaks at an early stage of the simulations and a new hydrogen bond between K489-NZ and G487-O is formed.

After MD simulations and conversion of Amber trajectories to xtc files (using $\mathrm{VMD}^{6}$ plugin "Topotools") the structural clustering was performed by employing GROMACS $4.5^{8}$ g_cluster tool and linkage method. Cluster analysis of the structure of parent iLOV and iLOV-Q489K along MD trajectories was carried out based on atoms positions in the side-chain of the FMN and residues in FMN 
binding pocket (i.e. Q489, N468, N458 and Q430). Each structure is added to a cluster when its distance to any element is less than a given cutoff. Clusters were obtained with a cut off value of $1.35 \AA$ and each cluster has a representative structure. The clustering process also calculates a middle structure for every single cluster obtained. After visualization and inspection, the representative structures from the highly clustered conformations of MD simulation trajectories were selected to be used in $Q M / M M$ geometry optimizations. 


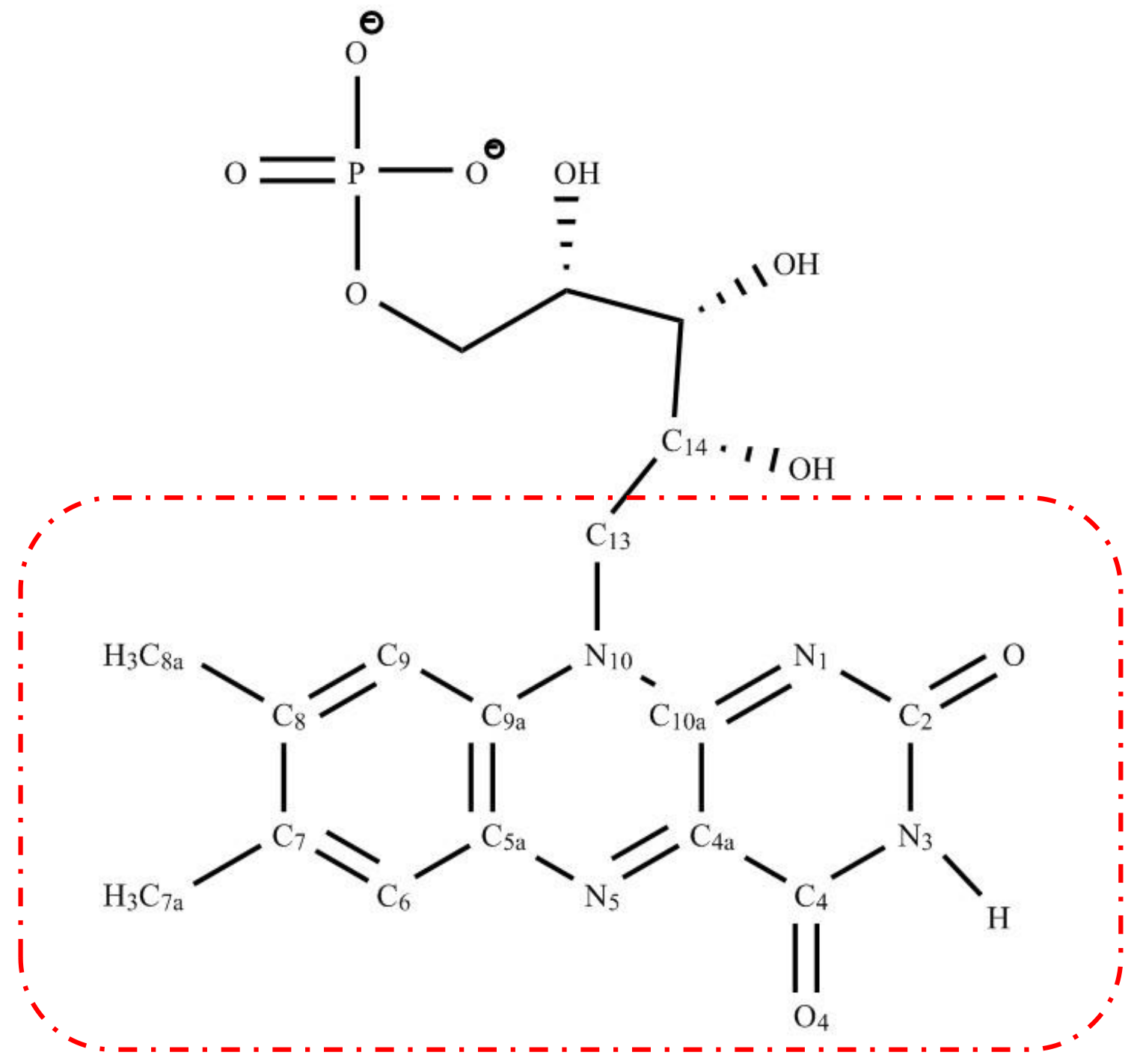

Figure S5: A perspective view of chemical structure and standard atom numbering system of flavin mononucleotide (FMN) used in this work. The QM region consists of the lumiflavin moiety of the FMN chromophore (in red rectangle). 
Table S2: Atomic charges of flavin mononucleotide (FMN), atom numbering system. RESP atomic partial charges calculated at HF/6-31G(d) level of theory for FMN used in this study; standard FMN atom numbering system is given in Figure S5.

\begin{tabular}{|c|c|c|c|c|c|}
\hline $\begin{array}{l}\text { Atom in } \\
\text { topology }\end{array}$ & \begin{tabular}{|c|} 
Standard \\
atom \\
numbering
\end{tabular} & $\begin{array}{c}\text { Charge } \\
\text { (a.u.) }\end{array}$ & $\begin{array}{l}\text { Atom in } \\
\text { topology }\end{array}$ & $\begin{array}{c}\text { Standard } \\
\text { atom } \\
\text { numbering }\end{array}$ & $\begin{array}{c}\text { Charge } \\
\text { (a.u.) }\end{array}$ \\
\hline N1 & $\mathrm{N} 1$ & -0.712596 & N4 & N10 & 0.009005 \\
\hline C1 & C2 & 0.914957 & C12 & C10a & 0.324135 \\
\hline O1 & $\mathrm{O}$ & -0.645631 & C13 & & -0.15906 \\
\hline N2 & N3 & -0.727744 & $\mathrm{H} 10$ & & 0.116816 \\
\hline $\mathrm{H} 1$ & & 0.400829 & $\mathrm{H} 11$ & & 0.116816 \\
\hline $\mathrm{C} 2$ & $\mathrm{C} 4$ & 0.712833 & C14 & & 0.371453 \\
\hline $\mathrm{O} 2$ & $\mathrm{O} 4$ & -0.622976 & $\mathrm{H} 12$ & & 0.073234 \\
\hline C3 & $\mathrm{C} 4 \mathrm{a}$ & 0.215833 & O3 & & -0.725951 \\
\hline N3 & N5 & -0.520759 & $\mathrm{H} 13$ & & 0.404832 \\
\hline $\mathrm{C} 4$ & $\mathrm{C5a}$ & 0.501029 & C15 & & 0.249754 \\
\hline C5 & C6 & -0.404743 & $\mathrm{H} 14$ & & 0.149577 \\
\hline $\mathrm{H} 2$ & & 0.240491 & $\mathrm{O} 4$ & & -0.880607 \\
\hline C6 & C7 & 0.064267 & $\mathrm{H} 15$ & & 0.550519 \\
\hline $\mathrm{C7}$ & $\mathrm{C} 7 \mathrm{a}$ & -0.196943 & C16 & & -0.148987 \\
\hline H3 & & 0.068423 & $\mathrm{H} 16$ & & 0.054279 \\
\hline $\mathrm{H} 4$ & & 0.068423 & O5 & & -0.475628 \\
\hline $\mathrm{H} 5$ & & 0.068423 & $\mathrm{H} 17$ & & 0.329456 \\
\hline C8 & $\mathrm{C} 8$ & 0.168603 & C17 & & 0.458665 \\
\hline C9 & $\mathrm{C} 8 \mathrm{a}$ & -0.197184 & H18 & & -0.023839 \\
\hline $\mathrm{H} 6$ & & 0.078595 & H19 & & -0.023839 \\
\hline $\mathrm{H} 7$ & & 0.078595 & O6 & & -0.731561 \\
\hline $\mathrm{H} 8$ & & 0.078595 & $\mathrm{P} 1$ & & 1.474459 \\
\hline C10 & $\mathrm{C9}$ & -0.396608 & O7 & & -0.983812 \\
\hline $\mathrm{H} 9$ & & 0.293011 & O8 & & -0.983812 \\
\hline C11 & C13 & -0.089815 & O9 & & -0.983812 \\
\hline
\end{tabular}




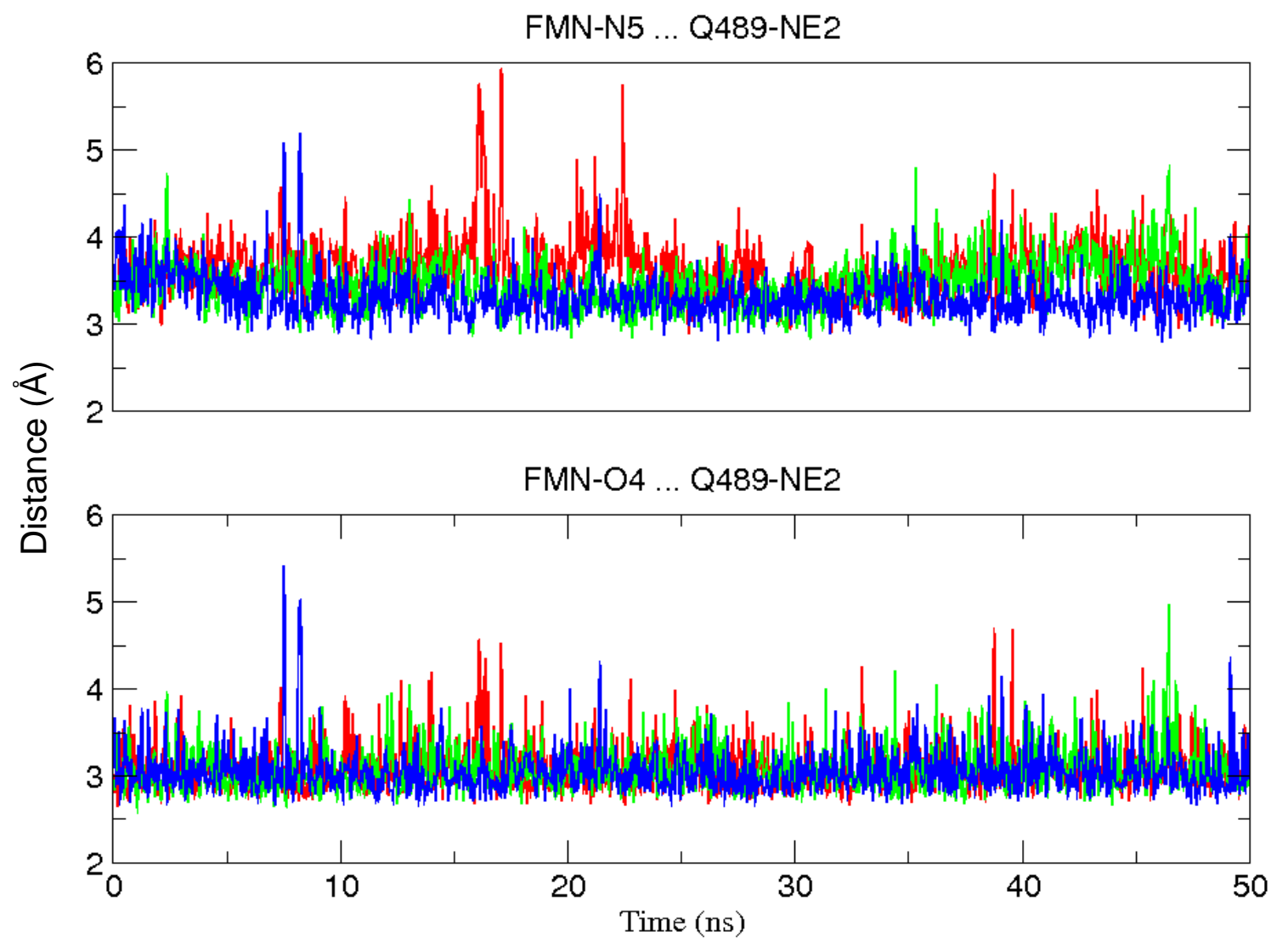

Figure S6: Interatomic distances for the indicated residues (atoms) pairs (in $\AA$ ) calculated over the MD trajectories of parental iLOV; (a) FMN-N5 and Q489NE2 distance, (b) FMN-O4 and Q489-NE2 distance for three independent MD runs (run_1, black, run_2, red, run_3,green). H-bond analysis in Table S3 shows that Q489-NE2 keeps a hydrogen bond with FMN-O4 during the simulations. 

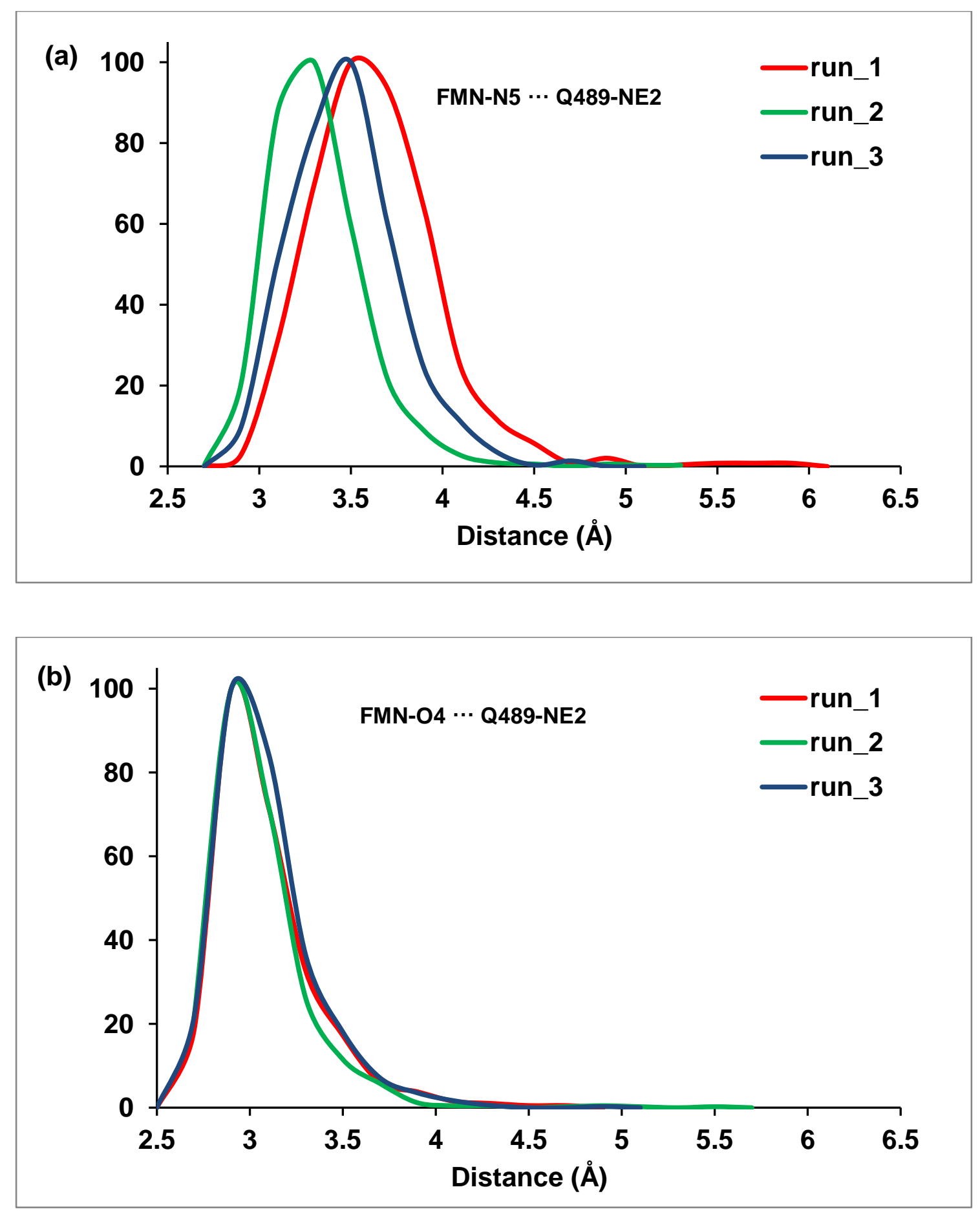

Figure S7: Distance distribution curve of the interatomic distances for the indicated residues (in $\AA$ ) calculated over the three independent MD trajectories of parental iLOV; FMN-N5 and Q489-NE2 distance, FMN-O4 and Q489-NE2 distance are shown. Q489-NE2 keeps a hydrogen bond with FMN-O4 during the simulations. 
FMN-N5 ... K489-NZ

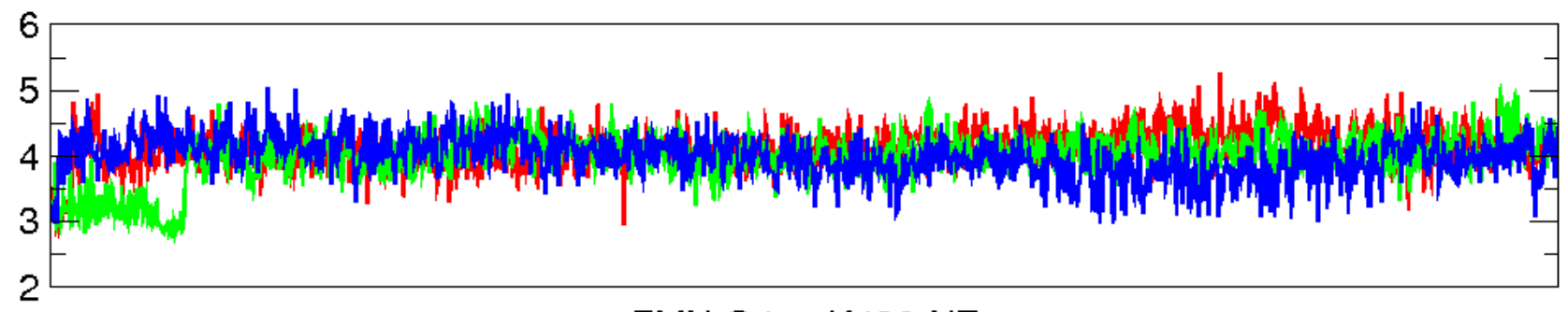

FMN-O4 ... K489-NZ
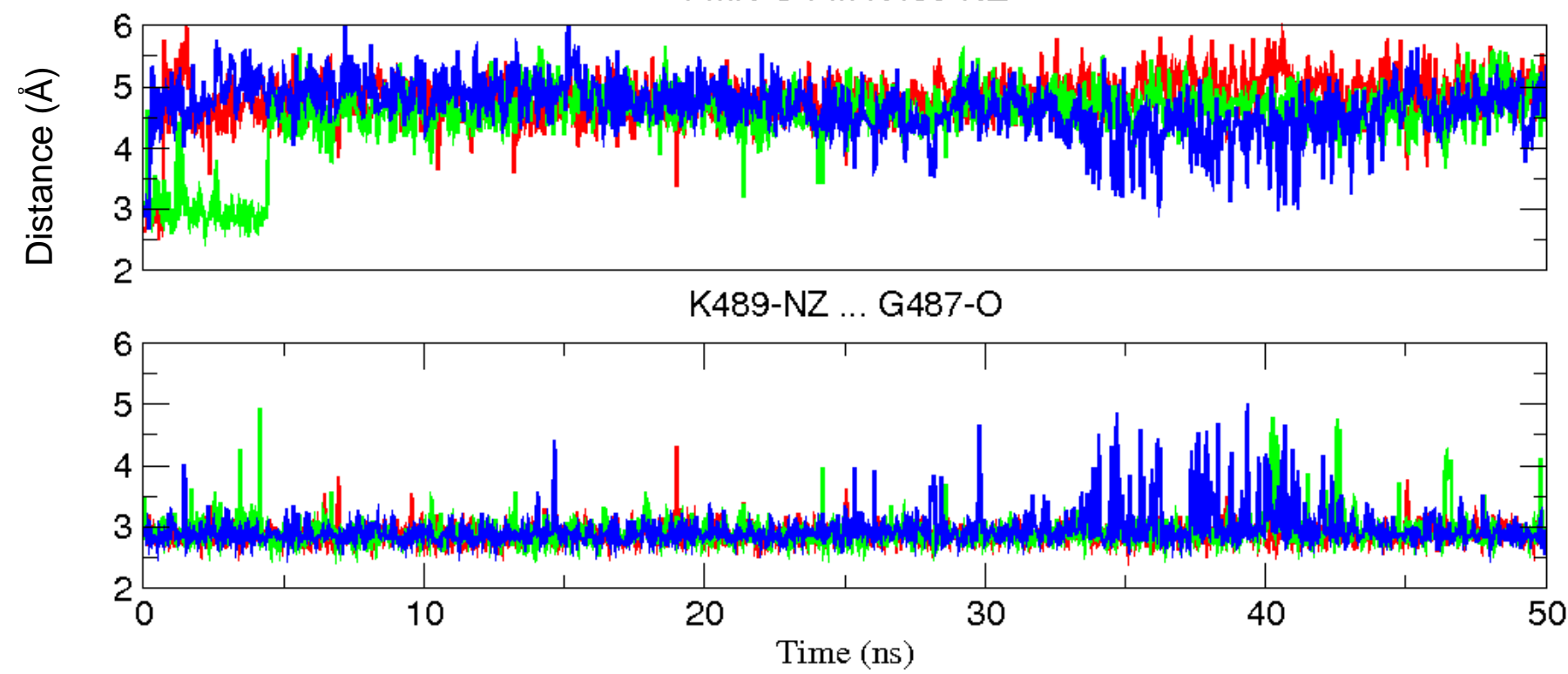

Figure S8: Interatomic distance for the indicated residues (in $\AA$ ) calculated over the MD trajectories of iLOV-Q489K variant; (a) FMN-N5 ․ K489-NZ distance, (b) FMN-O4 … K489-NZ distance, (c) K489-NZ and G487-O distance for three independent MD runs (run_1, black, run_2, red, run_3, green). K489 out conformation is the most populated conformation. 

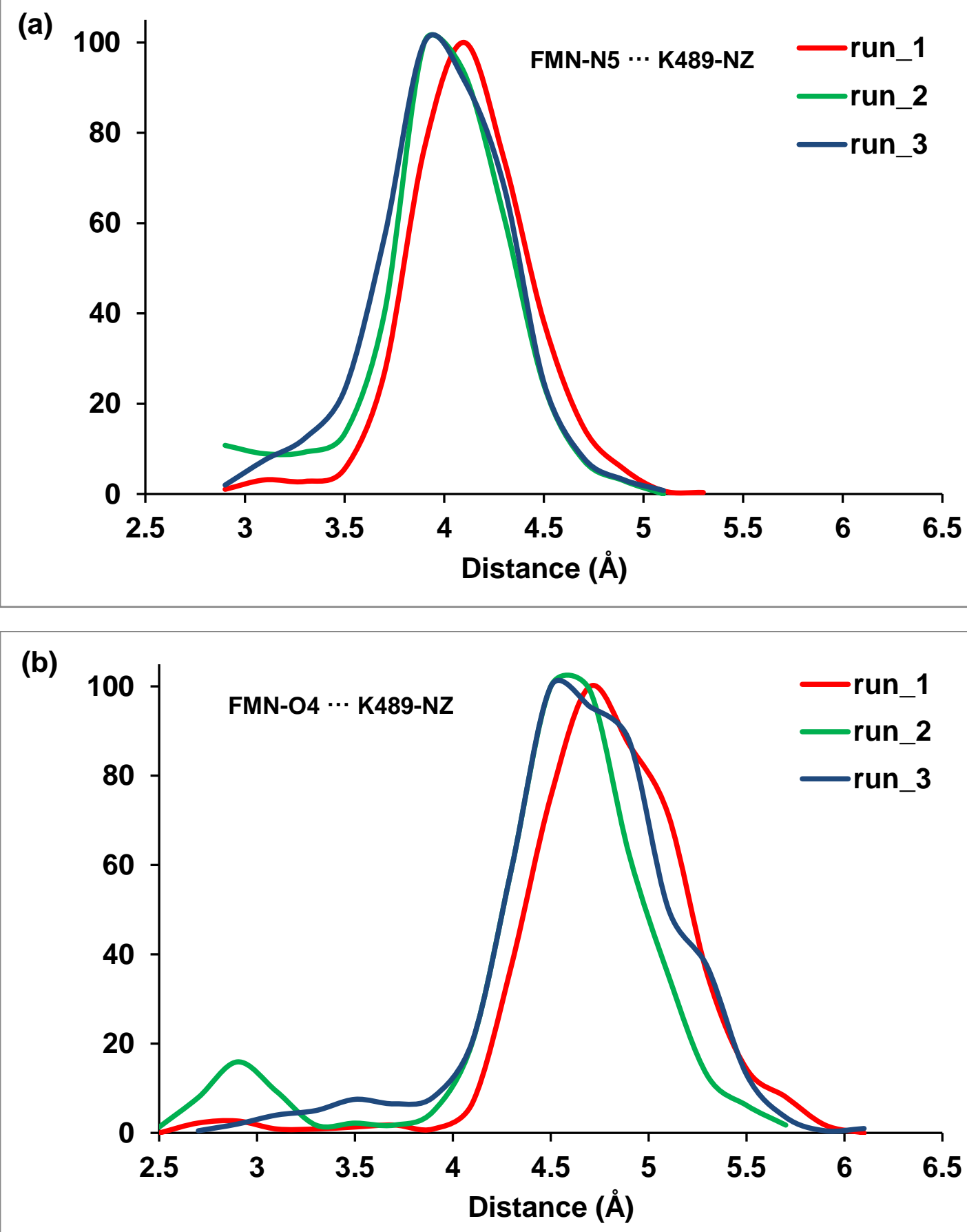




Figure S9: Distance distribution curve of the interatomic distances for the indicated residues (in $\AA$ ) calculated over the MD trajectories of iLOV-Q489K variant; (a) FMN-N5 … K489-NZ distance, (b) FMN-O4 … K489-NZ distance, (c) K489-NZ and G487-O distance for three independent MD runs (run_1, black,

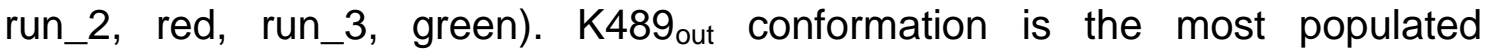
conformation. 
Table S3: Hydrogen bond analysis for (a) parental iLOV and (b) iLOV-Q489 variant along three independent MD trajectories $(3 \times 1000$ frames). Geometric distance and angle criteria for hydrogen bond identification were chosen as 3.2 $\AA$ and $150^{\circ}$, respectively. Average hydrogen bond distances (Avg. Dis.) and angles (Avg. Ang.) are also given.

\begin{tabular}{|c|c|c|c|c|c|c|c|}
\hline (a) & Acceptor & Donor H & Donor & Frames & Fraction & $\begin{array}{c}\text { Avg. Dist. } \\
(\AA)\end{array}$ & $\begin{array}{c}\text { Avg. Ang. } \\
\left({ }^{\circ}\right)\end{array}$ \\
\hline \multirow{2}{*}{ run_1 } & FMN-O4 & Q489-HE22 & Q489-NE2 & 686 & 0.686 & 2.96 & 164.9 \\
\cline { 2 - 8 } & FMN-N5 & Q489-HE22 & Q489-NE2 & 1 & 0.001 & 3.03 & 152.8 \\
\hline \multirow{2}{*}{ run_2 } & FMN-O4 & Q489-HE22 & Q489-NE2 & 699 & 0.699 & 2.95 & 164.1 \\
\cline { 2 - 8 } & FMN-N5 & Q489-HE22 & Q489-NE2 & 2 & 0.002 & 3.16 & 155.9 \\
\hline \multirow{2}{*}{ run_3 } & FMN-O4 & Q489-HE22 & Q489-NE2 & 680 & 0.68 & 2.96 & 164.9 \\
\cline { 2 - 8 } & FMN-N5 & Q489-HE22 & Q489-NE2 & 1 & 0.001 & 3.14 & 154.5 \\
\hline
\end{tabular}

\begin{tabular}{|c|c|c|c|c|c|c|c|}
\hline (b) & Acceptor & Donor H & Donor & Frames & Fraction & $\begin{array}{l}\text { Avg. Dist. } \\
(\AA)\end{array}$ & $\begin{array}{c}\text { Avg. Ang. } \\
\left({ }^{\circ}\right)\end{array}$ \\
\hline \multirow{3}{*}{ run_1 } & G487-O & K489-HZ2 & K489-NZ & 292 & 0.292 & 2.86 & 163.3 \\
\hline & G487-O & K489-HZ1 & K489-NZ & 266 & 0.266 & 2.86 & 164.0 \\
\hline & G487-O & K489-HZ3 & K489-NZ & 241 & 0.241 & 2.86 & 162.5 \\
\hline \multirow{3}{*}{ run_2 } & G487-O & K489-HZ2 & K489-NZ & 262 & 0.262 & 2.86 & 162.7 \\
\hline & G487-O & K489-HZ1 & K489-NZ & 255 & 0.255 & 2.86 & 162.9 \\
\hline & G487-O & K489-HZ3 & K489-NZ & 236 & 0.236 & 2.86 & 162.9 \\
\hline \multirow{3}{*}{ run_3 } & G487-O & K489-HZ3 & K489-NZ & 251 & 0.251 & 2.87 & 163.8 \\
\hline & G487-O & K489-HZ1 & K489-NZ & 243 & 0.243 & 2.88 & 163.3 \\
\hline & G487-O & K489-HZ2 & K489-NZ & 231 & 0.231 & 2.88 & 163.3 \\
\hline
\end{tabular}




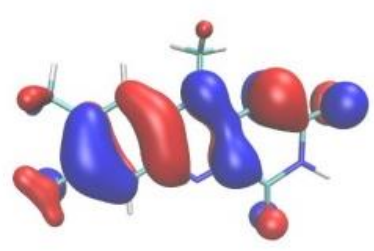

HOMO

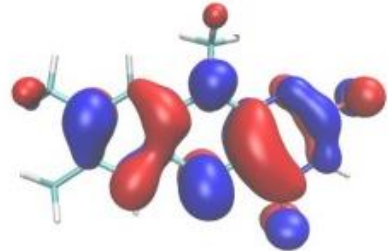

LUMO

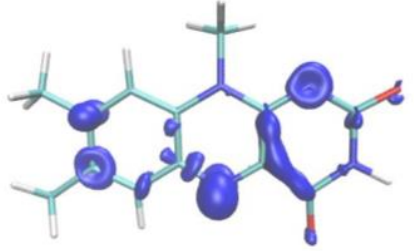

$\mathrm{S}_{0}-\mathrm{S}_{1}$ electron density difference

Figure S10: Frontier molecular orbitals (HOMO and LUMO) at the ground state geometry of FMN (modeled by LF) in parental iLOV using QM/MM TDB3LYP/SVP. S0-S1 electron density difference is also shown (isosurface $=0.025$ ). Examination of the molecular orbitals revealed that, for both parent iLOV and iLOV-Q489 variant, that the maximum excitation in the FMN model in the visible region of the spectrum is a $\pi-\pi^{*}$ transition. 
(a)

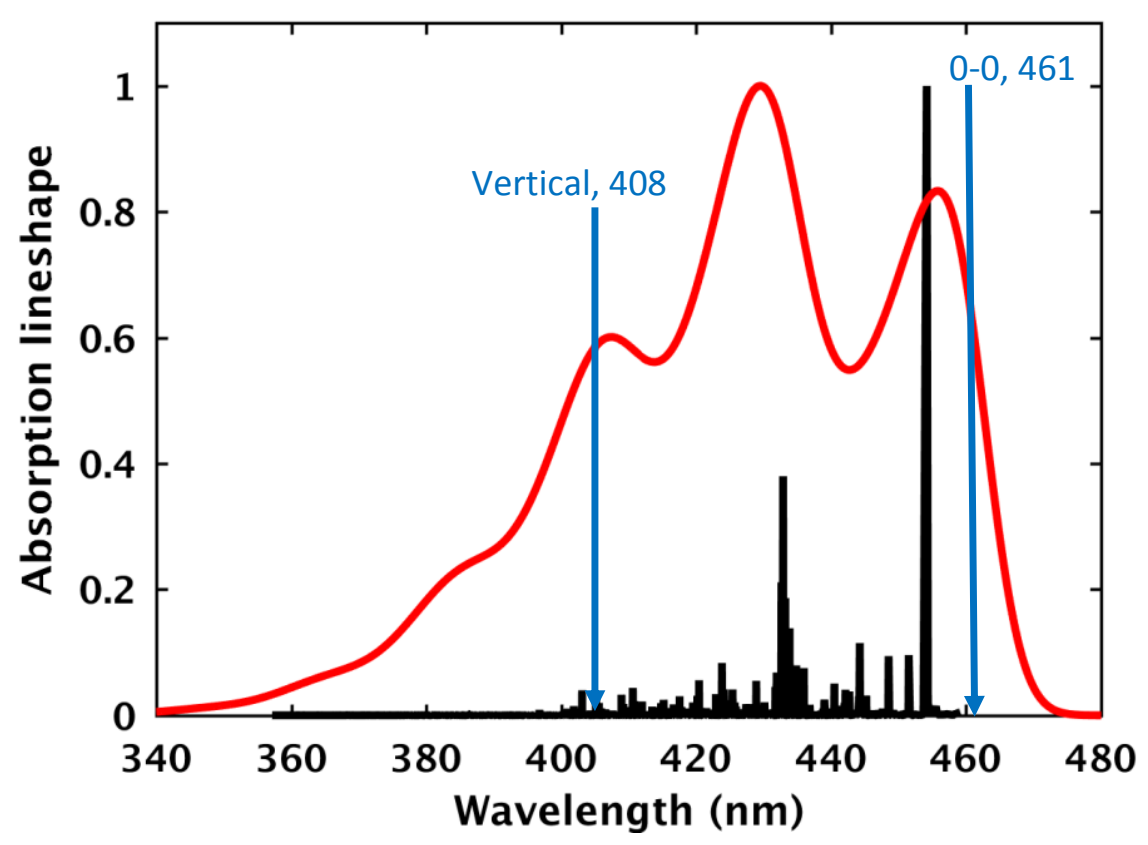

(b)

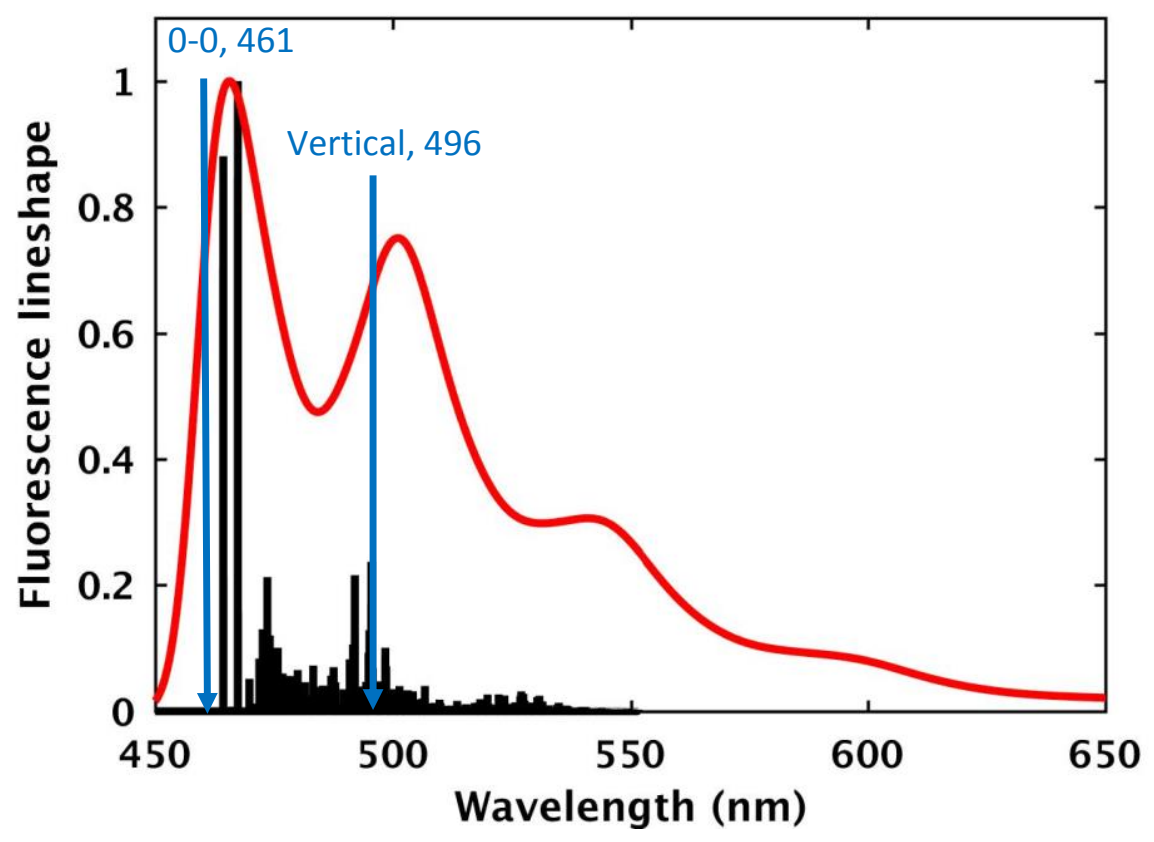

Figure S11: Vibrationally resolved (a) absorption and (b) fluorescence-emission spectra of FMN model lumiflavin calculated by using Adiabatic Hessian (AH) approach in the gas phase at $0 \mathrm{~K}$ calculated and using (TD) DFT B3LYP functional and the $6-31 \mathrm{G}(\mathrm{d})$ basis set; Gaussian convolution with $\mathrm{FWHM}=0.03$ $\mathrm{eV}$. Arrows mark the $0-0$ and vertical transition in excitation and fluorescence emission, respectively. Absorption and fluorescence-emission maxima are at 428 and $466 \mathrm{~nm}$, respectively. 
Table S4: Comparison of absorption and fluorescence emission of parental iLOV and iLOV-Q489K. Our determined experimental and theoretical absorption and fluorescence emission properties of parental iLOV and iLOV-Q489K are compared with the available literature data. The listed theoretical wavelength values for excitation and emission correspond to the vertical transition.

\begin{tabular}{|c|c|c|c|}
\hline system & $\lambda_{\max } \operatorname{exc}(\mathrm{nm})^{\star}$ & $\lambda_{\max } \mathrm{em}(\mathrm{nm})^{\star}$ & Reference \\
\hline parental iLOV & 447 & 497 & Christie et al. (experiment) ${ }^{3}$ \\
\hline $\begin{array}{c}\text { parental iLOV } \\
\text { (Q489 } \text { out }^{\text {(Q) }}\end{array}$ & 448 & 486 & Khrenova et al. (theory) ${ }^{9}$ \\
\hline $\begin{array}{c}\text { parental iLOV } \\
\left(\mathrm{Q} 489_{\text {in }}\right)\end{array}$ & 461 & 492 & Khrenova et al. (theory) ${ }^{9}$ \\
\hline iLOV-Q489K in $_{\text {in }}$ & 500 & 583 & Khrenova et al. (theory) \\
\hline parental iLOV & 449.5 & 496.5 & This work (experiment) \\
\hline iLOV-Q489K & 439.8 & 489.0 & This work (experiment) \\
\hline $\begin{array}{c}\text { parental iLOV } \\
\left(\mathrm{Q}_{\left.489_{\text {in }}\right)}\right.\end{array}$ & 414 & 486 & This work (theory) \\
\hline iLOV-Q489K in $_{\text {in }}$ & 440 & 534 & This work (theory) \\
\hline iLOV-Q489K out & 402 & 480 & This work (theory) \\
\hline
\end{tabular}

*The listed theoretical wavelength values for excitation end emission correspond to the S0,min-> S1 and S1, min-> S0 vertical transitions calculated by QM/MM TD-DFT method, respectively. The experimental values refer to the observed excitation and fluorescence emission maxima. 


\section{References}

1. Kleywegt, G. J.; Harris, M. R.; Zou, J. y.; Taylor, T. C.; Wählby, A.; Jones, T. A., The Uppsala electron-density server. Acta Crystallographica Section D: Biological Crystallography 2004, 60 (12), 2240-2249.

2. Schrodinger, L., The PyMOL molecular graphics system, version $1.3 \mathrm{r1}$. 2010. There is no corresponding record for this reference 2010.

3. Christie, J. M.; Hitomi, K.; Arvai, A. S.; Hartfield, K. A.; Mettlen, M.; Pratt, A. J.; Tainer, J. A.; Getzoff, E. D., Structural tuning of the fluorescent protein iLOV for improved photostability. Journal of Biological Chemistry 2012, 287 (26), 22295-22304.

4. Guerois, R.; Nielsen, J. E.; Serrano, L., Predicting changes in the stability of proteins and protein complexes: a study of more than 1000 mutations. Journal of molecular biology 2002, 320 (2), 369-387.

5. Schrodinger, L., The PyMOL molecular graphics system, version 1.3 r1. 2010.

6. Humphrey, W.; Dalke, A.; Schulten, K., VMD: visual molecular dynamics. Journal of molecular graphics 1996, 14 (1), 33-38.

7. Case, D.; Berryman, J.; Betz, R.; Cerutti, D.; Cheatham, I.; Darden, T.; Duke, R.; Giese, T.; Gohlke, H.; Goetz, A., AMBER 2015. University of California, San Francisco 2015.

8. Hess, B.; Kutzner, C.; Van Der Spoel, D.; Lindahl, E., GROMACS 4: algorithms for highly efficient, load-balanced, and scalable molecular simulation. Journal of Chemical Theory and Computation 2008, 4 (3), 435-447.

9. Khrenova, M. G.; Nemukhin, A. V.; Domratcheva, T., Theoretical characterization of the flavin-based fluorescent protein iLOV and its Q489K mutant. The Journal of Physical Chemistry B 2015, 119 (16), 5176-5183. 